\title{
ENVIRONMENTAL EXPOSURES
}

P Vineis, $H$ Ahsan, M Parker

Occup Environ Med 2005;62:657-662. doi: 10.1136/oem.2004.019190

A theoretical and practical discussion took place some years ago about the selection of high risk groups for the implementation of preventive activities. Particularly important in this discussion was Geoffrey Rose's seminal work. ${ }^{1}$ In this work, Rose set out the main advantages and disadvantages of such a "high risk group" preventive strategy. In Rose's words, it is a strategy with some clear and important advantages:

- The "high risk" strategy produces interventions that are appropriate to the particular individuals identified and consequently has the advantage of enhanced subject motivation.

- The "high risk" approach also offers a more cost effective use of limited resources and a more favourable ratio of benefits to risks.

Despite these advantages, the "high risk" strategy of prevention has some serious disadvantages and limitations. Firstly, as in all screening one is likely to meet problems with compliance, and the tendency is for the response to be greatest among those who are often least at risk of the disease; this, however, may be true for voluntary exposures, but not necessarily for occupational exposures. A second disadvantage is that this strategy is palliative and temporary. It does not seek "to alter the underlying causes of the disease but rather to identify individuals who are particularly susceptible to those causes". ${ }^{1}$ There is another, third, related reason why the predictive basis of the "high risk" strategy of prevention could be weak. It is well illustrated by data which relate breast cancer to parity and other reproductive factors. High risk women generate a relatively small proportion of the cases, too few to justify pre-screening for the identification of high risk women to whom to offer mammography. The lesson from this example is that a large number of people at a small risk may give rise to more cases of disease than the small number who are at a high risk. This situation seems to be common, and it limits the utility of the "high risk" approach to prevention. Combined with the first disadvantage mentioned-that is, the fact that those who are most likely to change their behaviour or seek treatment are often those at least risk, this implies that the effectiveness of the high risk strategy as a public health measure may be significantly lower than expected.

When Rose had these thoughts, the field of gene-environment interactions was in its infancy, at least for major chronic diseases. This meant that the implications of Rose's approach for screening for low-penetrance genetic susceptibility due to high -prevalence polymorphisms, were not explored at the time. It is to this issue that we turn in this paper.

\section{A PUBLIC HEALTH FRAME: NUMBERS NEEDED TO SCREEN}

To address the relevance of gene-environment interactions and genetic screening in the workplace we will use a framework we have described in more detail elsewhere. ${ }^{2}$ To assess the role of a gene-environment interaction and screening in a population we need to know the penetrance of the genetic trait (variant allele) and its frequency. Penetrance refers to the absolute risk of disease among individuals carrying the gene variant. So, high penetrance means high individual risk, and tends to be a feature of rarer gene variants or mutations. A useful measure for assessing the utility of an intervention is to estimate the number needed to treat (NNT) to prevent an event, which is equal to the inverse of absolute risk reduction. If screening is applied before intervening, NNT and frequency may be combined to compute what is called the number needed

See end of article for authors' affiliations

Correspondence to: Prof. P Vineis, Dept of Epidemiology and Public Health, Imperial College of Science, Technology and Medicine, Norfolk Place, London W2 IPG, UK: p.vineis@imperial.ac.uk to screen (NNS) to prevent one case of, say, cancer. In our previous paper ${ }^{2}$ we calculated the NNS in high risk families for a high-penetrant gene (BRCAl). The cumulative (lifetime) risk of breast cancer is around $80 \%$ in mutation carriers (at least in some studies and in some populations), and the frequency of mutations in high risk families is about $50 \%$. We supposed that tamoxifen or raloxifene halve the risk, based on results from randomised trials. Thus, we calculated that we would need to treat $2.5(1 /[0.8 \times 0.5])$ mutation carriers and screen $5(1 /[0.8 \times 0.5 \times 0.5])$ family members to prevent one cancer, under those circumstances. ${ }^{2}$ However, we also showed that if the general population were screened, the NNS changes greatly. Now the cumulative risk in mutation carriers is $40 \%$, with an absolute risk reduction by tamoxifen or raloxifene of $20 \%$, which means a number needed to treat of five mutation carriers. However, since only $0.2 \%$ of the general 
population are mutation carriers, the NNS is 2500 to prevent one cancer. One might discuss whether this large NNS would make BRCAl a realistic marker for use in the general population for screening. However, with dwindling cost and ease of high throughput genotyping technology this calculus may change in future, at least among certain populations/ families known to be at higher risk of carrying mutations.

Along the same lines, table 1 shows the calculations for a low penetrant gene (GSTMl), for which workers exposed to polycyclic aromatic hydrocarbons (PAH) are hypothetically screened. The example selected is one in which workers are screened for the GSTMl null genotype, and prevention subsequently focused on those testing "null" (for example, not hiring them in jobs that exposed them to $\mathrm{PAH}$ ). What would be the advantage of such an approach? From a review, the relative risk of lung cancer associated with the GSTMI null genotype is 1.34. Therefore, if the cumulative risk of lung cancer in workers exposed to PAH is $10 \%$, it will be about $13 \%$ in GSTMl null carriers. If the preventive intervention has a $50 \%$ effectiveness the cumulative risk after intervention would be $6.5 \%$, with a number needed to treat of 15 to prevent one cancer case. Since $50 \%$ of the population are carriers of the null genotype, we would need to screen 30 workers to prevent one cancer (table 1). Without screening workers for the GSTMl genotype, on the other hand, we would need to address prevention to 35 workers to prevent one cancer, a weighted average of the NNS among those testing positive and those testing negative for GSTMI (or, the sum of 15 needed to treat among those testing positive and 20 among those testing negative). This suggests that there would be little advantage in screening for the GSTMl gene, given that the NNS only decreases from 35 to 30. In other words, we would need to reduce the PAH exposure of 35 workers to prevent one cancer, in the absence of genetic screening, and we would have to reduce the PAH exposure of 30 workers if we did so through the identification, by genetic screening, of those "more susceptible" - a very modest achievement due to the low penetrance of the gene.

Simulation analysis has been used to calculate how many prospective workers for jobs with benzene exposure would have to be screened for CYP2El activity and $\mathrm{NAD}(\mathrm{P}) \mathrm{H}-$ quinone oxidoreductase (NQ01) alleles to find 1000 people without the known susceptible polymorphisms. In this study, the authors established that 2500 workers would need to be screened to hire 1000 genetically normal workers and thus prevent one case of benzene induced cancer. ${ }^{3}$

In another simulation study, Bartell and colleagues showed that genetic screening for chronic beryllium disease with HLA-DPBI*0201 gave health benefits that outweighed

\begin{tabular}{|c|c|}
\hline Measures & Estimates \\
\hline Relative risk & 1.34 \\
\hline Cumulative risk & $13 \%$ \\
\hline Risk reduction & $50 \%$ \\
\hline Cumulative risk after intervention & $6.5 \%$ \\
\hline Absolute risk reduction & $6.5 \%$ \\
\hline NNT & 15 \\
\hline Allele frequency (null genotype) & $50 \%$ \\
\hline NNS & 30 \\
\hline
\end{tabular}

financial costs only if avoidance of one case of the disease is valued at US\$1 million or higher. ${ }^{4}$ However, their estimate of predictive value might have been unrealistically high and might not have correctly weighed the harmful effects of falsepositive results.

Concerning false-positive results, one of the problems that epidemiological research is currently facing is the increasing number of associations among genetic variants, environmental exposures, and disease (gene-environment and genegene interactions). A very large number of such associations have been reported and, even more, are expected to emerge in the future. Tens of thousands of single nucleotide polymorphisms (SNPs) are or will be investigated in association with different diseases, and many of the observed associations will be false positives. The challenge is to distinguish false from true positives. A way to address this problem could be a Bayesian approach, based on the estimation of a prior probability and the calculation of posterior probability. The prior probability can be represented by previous studies, or biochemical or molecular information (for example, gene expression) that supports the function of an SNP, or other types of evidence such as alignment-conservation information. However, in the absence of such a systematic evaluation of results, premature transferral of genetic testing into practice could become a serious problem.

\section{ETHICAL ISSUES}

In addition to the advantages and disadvantages of genetic screening for low penetrance genes discussed above, the use of such screening, even were this known, in a particular case, to be effective as a public health measure, would also raise a number of important ethical and social issues which need to be taken account of in any decision about implementation. We will limit our analysis to screening of workers but similar considerations apply to environmentally exposed populations.

\section{Arguments in favour of the availability of genetics testing in the workplace}

There are some ethical arguments which might be drawn on by those who would favour the use, or at least the availability, of genetic screening of workers. Perhaps the strongest of these draws its strength from a long standing belief that employers and indeed legislators, have a duty, where this is possible, to protect employees, particularly those who are vulnerable, from avoidable risks in the workplace. Duties of this kind have been enshrined in employment legislation in the United Kingdom and many other countries for well over a century, for example, the Factory Act 1851 (outlawing child labour under the age of 8 ), and the Mines Act 1842 (outlawing women, girls and boys under 10 working in mines).

If one accepts that employers do indeed have a duty of care to their employees it might be reasonable to argue that where a test or screen is known to be effective, employers have an obligation to use it to improve the safety of workers and potential workers. This also suggests, in the legal context, that where such tests or screens do exist, but are not used, such employers may be vulnerable to legal challenge. Indeed such a case has recently occurred in the United States where the Dow Chemical Company was sued by the widow of a deceased employee for failure to include the employee in a cytogenetic testing programme which might have detected his development of leukaemia from exposure in the work- 
place to benzene at an early stage. ${ }^{5}$ However, this example does not refer to a genetic test for susceptibility, but to an intermediate marker; in addition, there is no sound evidence that cytogenetic markers in fact predict the onset of leukaemia accurately and that screening improves prognosis.

A second set of ethical arguments in favour of the availability of screening or testing arises out the broad duty of respect for freedom of choice (or autonomy). One might, for example, argue on this basis that making an informative test available, either commercially or in the workplace, would enable workers to make informed choices about the kinds of jobs they take-about whether or where to work. In at least one legal jurisdiction this right has been established legally. In the case of International Union UAW v Johnson Controls Inc, it was decided that the choice of whether or not to work in a hazardous environment, while pregnant in this case, was reserved for each individual to make themselves and was not for their potential employers to decide. ${ }^{6}$

Freedom of choice arguments claim that to deny workers access to informative tests is unacceptably paternalistic and is, potentially, either to condemn those who are at high risk to working in dangerous conditions, detrimental to their health unbeknownst to themselves, or to deny those who choose to do so, the opportunity to work where they wish.

One concern that has been raised in this respect is that the creation of a situation in which workers were free to use tests but employers were not would lead to "adverse selection" that is, where employees knew about their risks but employers, as insurers, did not and employees used this asymmetry to their advantage. This might be particularly a concern in contexts, such as the United States, where healthcare insurance is related to employment. Some have argued that this possibility supports "genetic transparency" where both parties should have access to such information. ${ }^{8}$

A third argument that might be used to support the use of genetic screening or testing in employment, in at least some situations, arises where this has the potential to be in the broader "public interest". One might imagine a situation in which the genetic screening of employees might be of relevance to public safety. An example might be screening those who are to be responsible for flying planes or working in air traffic control for mutations conferring a low risk of heart failure on the grounds that while unlikely, the occurrence of such failure would have serious implications for public safety.

A fourth and final argument in favour of the use of genetic screening in the workplace might be that this has the potential to bring about important economic advantages through increased safety and reduced healthcare costs. Again, this might be of particular relevance to companies operating in a country such as the United States where health insurance is tied to employment. But, taking economic advantage in the broader sense, such an argument might also be made in the context of countries with publicly funded health care.

\section{Arguments against the use of genetic testing in the workplace}

Despite these arguments in favour of the availability and use of genetic screening in the workplace under certain conditions, there are a number of important arguments against this which provide grounds for caution and, indeed, anxiety.

Possibly the strongest argument against the use of genetic testing in employment is that it has the potential to lead to increased discrimination. There is indeed, good evidence that this is already happening. Recently, for example, the US Equal Employment Opportunity Commission filed suit against the Burlington Northern Santa Fe Railroad Co. for defying the "Americans with Disability" Act (case settled in 2002 for US $\$ 2.2$ million) on the grounds that the company required employees to submit blood samples to test them for genes predisposing to the carpal tunnel syndrome. It was also argued, successfully, that the company failed to obtain adequate informed consent and in some cases threatened employees with dismissal for failing to comply. ${ }^{7}$ This is one among many examples of such discrimination. ${ }^{5}$

In addition to discrimination against individuals, such as that exemplified by the case above, genetic screening in the workplace also brings with it the potential for discrimination against groups that come to be seen as "high risk":

"If one group is continually trumpeted in the media in association with a host of genetic diseases, [or vulnerabilities] members of the group may find themselves considered less desirable as mates and employees".

Discrimination might also arise out of the selective or partial use of genetic screening. Economic factors may lead to a temptation to use the tests less with highly skilled workers or those who are most needed and this will favour those already best off, or discriminate against those already most vulnerable; for example, if there is a shortage of people willing to working with a particularly dangerous process and testing is withheld in order to maintain a workforce.

In general then, arguments about discrimination arise out of a concern that genetic screening in employment may lead to a situation in which a person's genetic make up determines their opportunities. ${ }^{6}$ That is, to a situation in which those individuals who test "positive" may as a result become less employable, less insurable, and vulnerable in a number of different and important respects. Such concerns do not arise solely out of the nature of genetics or of genetic information but also out of the social and political realities of the world in which people live and work. Arguments about freedom of choice, for example, may sound attractive in the abstract, but policies based on freedom of choice divorced from an awareness of the broader social context have the potential to favour the wealthy, the highly educated, and the genetically healthy. Not everyone, for example, has the choice about where they are going to work-lack of skills, lack of mobility, living in an area of high unemployment, and so on may make it impossible for those who are rejected from local employment to find such employment elsewhere.

Secondly, in addition to its potential to lead to increased discrimination, the use of genetic screening in the workplace may lead to an increased likelihood of invasions of the privacy and confidentiality of workers; for example, in the writing of references, the provision of information for the purposes of insurance, and so on. Furthermore, the standards of security and confidentiality in relation to the use of genetic information and samples may be less rigorously monitored in the context of employment than in, for example, medical research. Indeed, examples already exist of samples being tested for outcomes other than that for which they were taken; for example, in the case of Norman-Bloodsaw $\mathrm{v}$ Lawrence Berkeley Laboratory employees provided blood and urine samples for cholesterol testing, but in fact some of 
these samples were subsequently tested for syphilis, pregnancy, and sickle-cell trait. ${ }^{6}$

A third set of arguments against the use of genetic screening for low penetrance genes in the workplace arises out of concerns that the information provided by such tests is likely to be extremely difficult to interpret and/or to communicate. Firstly, there is the question, explored in the first section of this paper, about the extent to which such testing is likely to produce information of any additional use in the workplace. Secondly, there is the question of how risk information, for example, in the case of low penetrant genes associated with a mild increase in cancer risk is to be communicated to employers and to employees in a way that is understandable or usable. Thirdly, there is a great deal of evidence that even in the case of single gene disorders where the mode of inheritance and risk are, by comparison, clear, those tested have a tendency to misunderstand the implications of test results, especially where these are negative. ${ }^{10}$

The fourth and final set of arguments against the use of genetic screening and testing in the workplace is that this is a distraction from the responsibility of employers and legislators to ensure that the working environment is safe for all of those who work there. Instead of using resources to identify workers who are less at risk, the focus should be on finding ways to make the workplace safe for all. The evidence from the first part of this paper suggests that in addition to its ethical importance, this argument has some scientific and health economic merit. Of course, quite similar arguments apply to environmentally exposed populations.

\section{AN EXAMPLE: OCCUPATIONAL AND ENVIRONMENTAL EXPOSURE TO ARSENIC}

We will consider a somewhat extreme example-that is, an exposure that is important both for industrial workers and for the general population in wide areas of the world. The example has been chosen because the identification of high risk groups could be extremely useful, at least in theory, to overcome the practical difficulties and the costs of primary prevention in affected populations.

Chronic exposure to arsenic is known to cause nonmelanocytic skin and internal tumours in humans. Exposure to arsenic commonly happens in occupational and environmental settings. Although occupational exposure to arsenic occurs in a variety of industrial settings, the predominant source of arsenic exposure for $>100$ million people worldwide, including $\sim 70$ million in Bangladesh and adjoining parts of India, has been from contaminated drinking water. ${ }^{11}$ Given the magnitude of the problem, which the WHO labelled as the largest mass poisoning event in human history, the issue of risk reduction of arsenic induced health problems became an important research and policy topic. Since millions of people already accrued chronic exposure, and their risk of cancer has increased several-fold, measures of secondary and even tertiary prevention also become pertinent in addition to primary prevention. The ability to isolate "high risk" groups would contribute enormously to the development of an effective preventive strategy. For this reason the knowledge of mechanisms of arsenic carcinogenesis can help. Several studies have examined the role of oxidative stress and DNA repair genes in the susceptibility to arsenic effect on premalignant skin lesions. In these studies, carriers of certain polymorphisms in oxidative stress genes myeloperoxidase (MPO) and catalase (CAT) and DNA repair gene xeroderma pigmentosum complementation group D
(XPD) have been shown to have 3-11-fold higher risk of premalignant skin lesions than the non-carriers. ${ }^{12}{ }^{13}$

Arsenic has a dose dependent effect on skin and internal tumours. The risk of cancer among arsenic exposed population is $\sim 1 \%$ but the risk for premalignant skin lesions is much higher, ranging from $<1 \%$ to $>10 \%$, depending on the dose and duration of exposure.

Let us make an extreme assumption-that is, that the relative risk for premalignant lesions is 11 (the highest estimate in literature), and that the cumulative risk of such lesions is $10 \%$. Also, let us assume: (a) that the intervention leads to a $50 \%$ decrease in the risk of skin lesions; or (b) that such decrease is $100 \%$. Clearly, these are unrealistic assumptions, since a relative risk of 11 is more compatible with a highly penetrant gene than with a low penetrant gene. Table 2 shows the theoretical calculations under such strong assumptions. The table should be interpreted in the following way: (1) we suppose we have an intervention (improving the quality of water) that reduces the risk of premalignant lesions by $50 \%$; in this case we have to "treat" 20 subjects with wild-type genotype (NNT) to prevent one lesion, given that the cumulative risk of lesions is $10 \%$ for the wild-type; (2) if our intervention is $100 \%$ effective, then the NNT is $10-$ that is, for every 10 treated persons (wild-type) we prevent a case; (c) if we screen for a gene that multiplies the risk of skin lesions by 11 (extreme estimate in the literature)-that is, a cumulative risk of $100 \%$ and the intervention is $50 \%$ effective, the NNT is 2 for those testing positive (that is, in every two people 1 is "saved"); but in fact to identify the subjects with the variant gene we need to screen the population: with a prevalence of $20 \%$ of the variant, the NNS becomes 10; (4) finally, with 100\% effectiveness, we still have to screen five to find the one that will be saved from skin lesions. (As in the example above, we can then compute the number needed to treat in the absence of screening, which is the weighted mean of the NNT among those testing negative and those testing positive-that is, 16.4 for $50 \%$ efficacy and 8.2 for $100 \%$ efficacy of the preventive effort). Is this worth it? Clearly not. Apart from the small differences between 20 and 10, or 10 and 5, the main argument is moral: cleaning the water for everybody will have a number of important implications in addition to preventing skin cancer.

It is clear that the most obvious and effective prevention strategy would be the reduction of exposure. If a high risk strategy is considered as an option, rather than genetic screening (because of uncertain and probably unfavourable NNT and NNS), identification of the at-risk population through screening for arsenic exposure of the population by testing drinking water and biological samples (urine, hair, or nail) for arsenic will be more practical. Since the distribution of arsenic exposure is somewhat less individual specific (unlike genetic polymorphisms), instead of genetically tailored, individual-level interventions, household-level interventions (provision of safe wells) may be more effective. For many arsenic affected areas where $50-90 \%$ of the population are exposed (for example, in Bangladesh, West Bengal, India, Inner Mongolia, and certain provinces of China) community-level interventions (for example, community wells, supply water, food fortification with antioxidants, or other anti-arsenic nutrients) may be warranted. Irrespective of the availability of specific pharmacological or nutritional interventions, identification of exposed population for avoidance of further exposure would be beneficial. 
Table 2 Calculation of the number needed to screen for a hypothetical highly penetrant gene among subjects exposed to arsenic

\begin{tabular}{|c|c|c|c|c|}
\hline & \multicolumn{4}{|l|}{ Gene } \\
\hline & \multicolumn{2}{|c|}{ Wild-type } & \multicolumn{2}{|c|}{ Variant } \\
\hline & (a) & (b) & (a) & (b) \\
\hline Relative risk for gene & 1.0 & 1.0 & 11 & 11 \\
\hline $\begin{array}{l}\text { Cumulative risk of } \\
\text { premalignant lesions }\end{array}$ & $10 \%$ & $10 \%$ & $100 \%$ & $100 \%$ \\
\hline Risk reduction \% & $50 \%$ & $100 \%$ & $50 \%$ & $100 \%$ \\
\hline $\begin{array}{l}\text { Cumulative risk after } \\
\text { intervention }\end{array}$ & $5 \%$ & 0 & $50 \%$ & \\
\hline Absolute risk reduction & $5 \%$ & $10 \%$ & $50 \%$ & $100 \%$ \\
\hline NNT & 20 & 10 & 2 & 1 \\
\hline Carrier frequency & $80 \%$ & $80 \%$ & $20 \%$ & $20 \%$ \\
\hline NNS & 25 & 12.5 & 10 & 5 \\
\hline & & (a) & (b) & \\
\hline $\begin{array}{l}\text { NNT in the absence of } \\
\text { screening }\end{array}$ & & 16.4 & 8.2 & \\
\hline
\end{tabular}

Two assumptions are made: that the preventive intervention has $50 \%$ (a) or $100 \%$ (b) efficacy (see text).

Finally, an ethical aspect that pertains to the issue of tailored screening for reducing health effects from arsenic in drinking water needs consideration. For many arsenic affected countries the major source of arsenic exposure is from drinking water-a basic element for which access to every individual is an individual right rather than a privilege. Therefore, provision of safe drinking water may be viewed as an obligation rather than as a research issue (it is a primary good in that it is a prerequisite for the "functioning" of citizens in society).

\section{CONCLUSIONS}

In this paper we have tried to show that, at least for low penetrant genes, screening for genetic variants in order to identify high risk subgroups in the population is not a practical and useful strategy, or at least we cannot find obvious examples. Even in an extreme case, arsenic pollution of wells in wide areas of developing countries, assuming the highest estimates for the strength of association between susceptibility genes and the risk of disease does not support genetic screening as a reasonable and moral approach to prevention.

\section{Authors' affiliations}

P Vineis, Imperial College London, UK, and University of Torino, Italy H Ahsan, Department of Epidemiology, Mailman School of Public Health, Columbia University, New York, NY, USA

M Parker, The Ethox Centre, Oxford University, UK

Competing interests: none declared

\section{REFERENCES}

1 Rose G. Sick individuals and sick populations. Int J Epidemiol 1985;14:32-8

2 Vineis $\mathbf{P}$, Schulte P, McMichael AJ. Misconceptions about the use of genetic tests in populations. Lancet 2001;357:709-12.

3 Nicas M, Lomax GPA. Cost-benefit analysis of genetic screening for susceptibility to occupational toxicants. J Occup Environ Med 1999;41:535-44.

4 Bartell SM, Ponce RA, Takaro TK, et al. Risk estimation and value-ofinformation analysis for three proposed genetic screening programs for chronic beryllium disease prevention. Risk Anal 2000;20:87-99.

5 Brandt-Rauf PW, Brandt-Rauf SI. Genetic testing in the workplace: ethical, legal and social implications. Annu Rev Public Health 2004;25:139-53.

6 Desmond J, Gardner-Hopkins JD. Unemployable genes: genetic discrimination in the workplace. Auckland University Law Review 2001;9:435-68
7 Human Genetics Commission. Inside Information: balancing interests in the use of personal genetic data. Human Genetics Commission, 2002.

8 Diver CS, Cohen JM. Genephobia: what is wrong with genetic discrimination? University PA Law Review 2001;149:1439-82.

9 Davis DS. Genetic research and communal narratives. Hastings Center Report 2004;34:40-9.

10 Michie S, Smith JA, Senior V, et al. Understanding why negative genetic test results sometimes fail to reassure. Am J Med Genet 2003;1 19:340-7.

11 Rahman MM, Chowdhury UK, Mukherjee SC, et al. Chronic arsenic toxicity in Bangladesh and West Bengal, India-a review and commentary. J Toxicol Clin Toxicol 2001;39:683-700.

12 Ahsan $\mathrm{H}$, Chen $\mathrm{Y}$, Wang $\mathrm{C}$, et al. DNA repair gene XPD and susceptibility to arsenic-induced hyperkeratosis. Toxicol Lett 2003;143:123-31.

13 Ahsan H, Chen Y, Kibriya MG, et al. Oxidative stress genes myeloperoxidase and catalase and susceptibility to arsenic-induced hyperkeratosis. Cancer Lett 2003;201:57-65.

\section{QUESTIONS (SEE ANSWERS ON P 597)}

(1) How would you define the number needed to screen?

(a) The number of subjects I need to screen to detect a case of disease

(b) The number of screened subjects whose lives can be saved by the screening procedure

(c) The number of subjects I need to screen to prevent a case of disease

(d) The number of subjects with disease that the screening procedure correctly identifies

(e) The number of subjects that need to undergo the screening procedure in a given population

(2) Identify two reasonable arguments in favour of genetic screening of workers:

(a) The right of the worker to know about her susceptibility to occupational diseases

(b) A case where the genetic screening of employees might be of relevance to public safety

(c) The need for providing appropriate (tailored) insurance to all workers

(d) The possibility of screening the most susceptible for early disease

(e) The possibility of selecting between potential employees

(3) Identify two important arguments against genetic screening of workers:

(a) Genetic screening can generate many false negatives and false positives, the management of which can create problems

(b) Genetic screening may lead to a situation in which an individual's genetic make up determines their opportunities of employment

(c) The prevalence of genetic susceptibility is usually too low to warrant screening procedures

(d) Genetic screening can be a distraction from the responsibility of employers to ensure that the working environment is safe for all

(e) Genetic screening is too expensive

(4) Identify two good arguments against screening of people in Bangladesh for genetic susceptibility to arsenic induced skin lesions:

(a) Too low occurrence of skin lesions

(b) Too low prevalence of genetic susceptibility

(c) It is an unethical approach to select the most susceptible individuals instead of providing safe water (a primary good) to all

(d) The NNS is not very far from the number of people who would benefit from the provision of safe water

(e) There is no evidence that genetic susceptibility interferes with the effects of arsenic in water

(5) Identify an important principle of prevention that underlies the choice of preventive strategies:

(a) A large number of subjects at high risk is needed to implement prevention 
(b) Primary prevention is more effective if it is tailored for a small number of high risk individuals

(c) The whole population is the best target for primary prevention, because the occurrence of disease is higher (d) A large number of people at a small risk may give rise to more cases of disease than the small number who are at a high risk

(e) A "high risk" strategy alters the underlying causes of the disease because it identifies individuals who are particularly susceptible to those causes 